\title{
Research on Experiential Layout of Amusement Space in Jiande Aviation Town
}

\author{
Wei Liu ${ }^{1 *}$, Yingzhi Liu ${ }^{1}$ and Qiuye Liu ${ }^{1}$ \\ ${ }^{1}$ School of Design \& Art, Shenyang Aerospace University, Shenyang, Liaoning, 110136, China
}

\begin{abstract}
The aviation town is a strong way to spread aviation culture and also a form to improve the attraction of a city, which has become the main focus of the development of the aviation tourism industry at present. This article took Jiande Aviation Town as an example and analyzed the experience of amusement space of Jiande Aviation Town from the standpoint of aviation leisure block and aviation service block including the experiential study of Jiande Aviation Town on innovation, value and competitiveness to arrival at a conclusion: Innovation determines value, which means innovation on tourists' experience way can effectively raise sense of mental satisfaction and sense of affirmation of tourists, and fully raise the value of aviation town; Innovation determines competitiveness, which means only by continuously innovating to satisfy tourists' experience, can we enhance the competitiveness of the industry.
\end{abstract}

\section{Jiande Aviation Town}

\subsection{Development status of the aviation town}

The development of aviation towns in China is still at a conceptual stage, and the direction of development is not clear that leads to the fragmentation of the development of the aviation towns[1]. The development of the aviation town should be based on the actual situation of the aviation industry, and at the same time, it should be constructed and designed in accordance with the cultural characteristics and the economic development of China. Building a "transportation power" is an important policy proposed by the 19th National Congress of the Communist Party of China. This is an extremely great development opportunity for the development of the aviation towns. Grasping the opportunity and actively catering to the development of national policies can promote the development of China's aviation industry and make the town maturer and stabler. Low-altitude tourism refers to the aviation tourism, sports and entertainment activities using aviation aircraft in the lowaltitude range, which plays an irreplaceable role in other traditional tourism modes in the tourism market and is an important manifestation of the cross-combination of the aviation industry and the tourism industry[2]. The aviation town has a good ecological environment and uses abundant resources to create a high-end tourism industry mainly based on aviation and combines a series of distinctive industries such as folk customs to create experiential tourism characteristic products[3]. The entire industrial chain such as the design, the development and the production of the aviation town industry can be linked with new high-end technologies such as the application of the "Internet + " model, the hottest big data technology and the widely used cloud computing[4]. These are applied to the experiential amusement projects to increase tourist experience, and the development of tourism products should transition from pure sightseeing tourism products to vacation experience products[5].

\subsection{Overview of Jiande Aviation Town}

Jiande Aviation Town is located in the west of Hangzhou City, Zhejiang Province. It is a well-known national aviation town with a planned area of more than $16,473.69$ acres in its core area. Jiande Aviation Town has become a leader in driving the economic development of Jiande City. The municipal government has made efforts to build Jiande Aviation Town into a multifunctional development zone integrating aviation, new energy, new functional materials, health preservation and building materials machinery. Jiande Aviation Town Project integrates traditional industries and emerging industries, which also realizes the organic combination of traditional models and emerging reforms. Jiande Aviation Town is the cradle of aviation professionals and a planning block for aviation education and training bases. The town has an excellent ecological environment and has tourism advantages. It combines ecological resources and folk customs to create a unique aviation town for tourists, which can relax and keep in good health, understand the local customs and nature also include aviation experience. Jiande Aviation Town construction is building a leading town in the entire aviation industry chain in China. Zhejiang Province puts the construction of Jiande Aviation Town in a prominent position. Starting from the development of aviation

* Corresponding author: ${ }^{*} 1424817759 @ q q . c o m$ 
operations, it has realized and paid equal attention to the development of theme parks and the development of the service industry and has successfully created a general aviation industry chain that belongs to Jiande Town. Jiande Aviation Town has been highly valued by the Zhejiang provincial government for several years and has successively won several national and local honors. Jiande Aviation Town actively responds to national

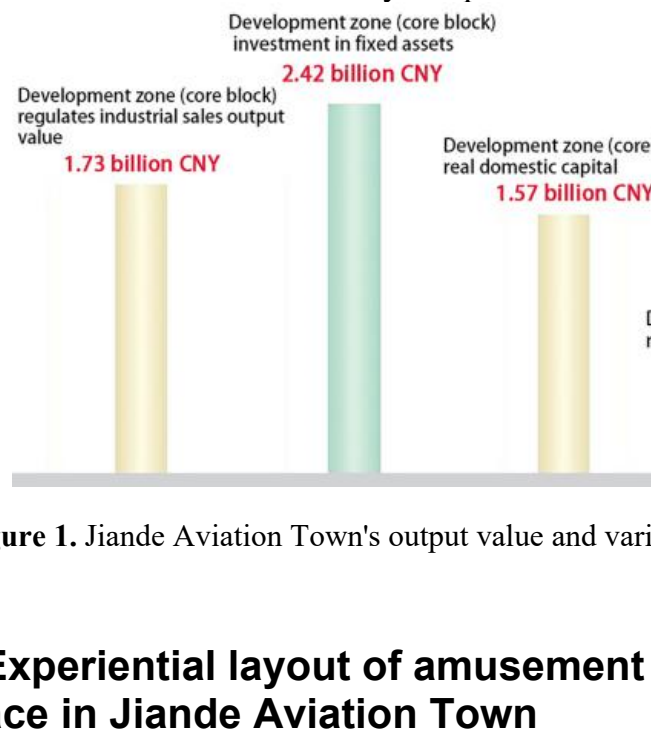

\subsection{General layout of Jiande Aviation Town}

Jiande Aviation Town includes the aviation leisure tourism sector and the aviation project services sector as well as the aviation knowledge and cultural technology sector. The master plan of the town puts forward the concept of "town is a scenic spot" and strives to create a scenic town with one scene by step. The aviation town resource planning is reasonable and forming an overall

\section{2 space in Jiande Aviation Town}

policies and has become a key aviation industry base in Zhejiang Province (Figure 1). It has established a wellknown characteristic aviation town in China as its development positioning and has realized the transition from an empty city to a lively city.
Development zone (core block) foreign investment 30 million USD

Development zone (core block) 33.32 million CNY

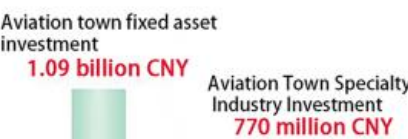

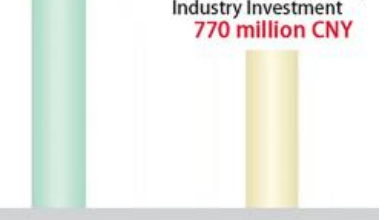

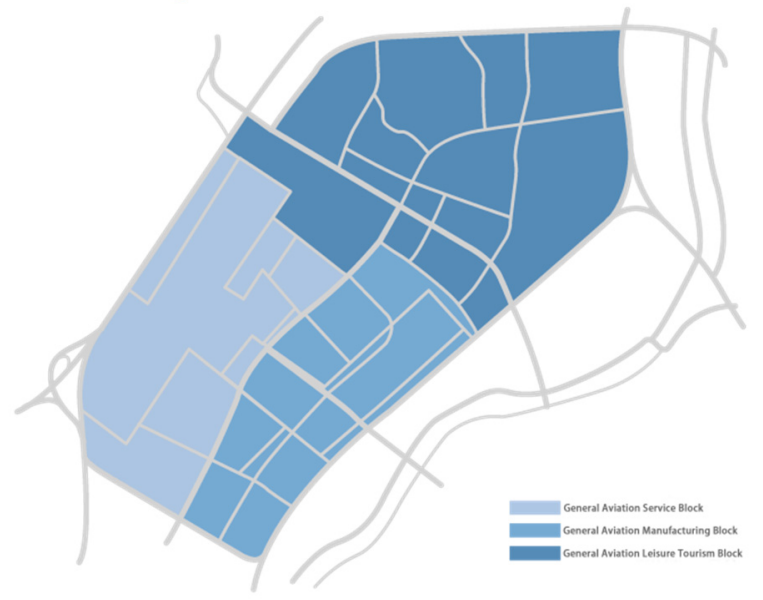

Figure 2. The three major blocks of Jiande Aviation Town (source: self-drawn by the author) 




Figure 3. The five major areas of Jiande Aviation Town (source: the self-drawn by the author)

\subsection{Aviation leisure travel experience block}

The leisure travel experience of Jiande Aviation Town requires that the aviation town must have the necessary facilities to meet the needs of tourists. These resources include infrastructure such as airplanes, parking aprons, repair shops and other facilities while also meeting the basic needs of tourists such as accommodation, travel and food. Jiande Aviation Town through the reasonable allocation of resources and the improvement of aviation infrastructure to enhance the tourist experience, so as to promote the innovative development of the aviation towns and improve the economic benefits of the aviation towns to achieve the purpose of low operations and high returns. Jiande Aviation Town has very high-quality human resources such as the former site of Hengshan Ferroalloy Factory and the surrounding industrial technical schools. The town transformed these unique resources into the aviation tourism resources to Jiande Aviation Town and created an aviation and leisure theme park with the theme "Aerospace" combining Henggang Industrial Site with surrounding industrial technical schools, Xin'an Jiang yu Hot Spring and urban-rural junction, which made people remember the past and feel the changes of the times, also can train new cultural skills and improve a "holy place" for personal ability. The aviation town combines hotel catering culture experience, amusement project experience, leisure health experience and low-altitude tourism experience into one, providing tourists with a charming and unforgettable aviation experience.

\subsubsection{Experience of low-altitude tourism and} skydiving. Low-altitude tourism is an emerging industry. Its advantages are reflected in the fact that it can not only meet the needs of tourists for the aviation flight experience but also provide tourists with the high-end tourism and air sightseeing services, which is an important manifestation of the cross-combination of the aviation industry and the tourism industry as a new model of "general aviation + tourism". In order to promote the supply-side reform of China's tourism industry and the innovation and development of the general aviation industry in various places, the state has focused on advancing the overall tourism strategy while expanding the tourism market[2] and has promulgated a lot of policies to support the low-altitude tourism industry. The low-altitude tourism project of Jiande Aviation Town provides tourists with an opportunity to take a plane tour to see the well-known national scenic spots such as the Fuchun River and Xin'an River in the sky while inspiring the patriotism. While overlooking the ancient villages in the south of China, such as Xinye Ancient Village, tourists will experience the humanistic feelings in the architectural community. The low-altitude sports events in the aviation town include gliding, hot air balloon, aviation models and other sports, which allow visitors to participate in the thrills or the pleasure of relax in the sky and overlook the earth. Combining the real flight experience with tourism, and leisure functions is the outstanding advantage of Jiande Aviation Town's amusement space. AirAsia's hot air balloon is also a lowaltitude tourism project provided by Jiande Aviation Town, which has highly ornamental. Tourists can experience the beauty of nature and the joy of life in a slowly rising hot air balloon. Some professionals introduce aviation knowledge to tourists while overlooking the beauty of the town so as to allow tourists to learn the relevant theoretical knowledge when they experience it, and put the wings of the theory onto the practice. The Hump skydiving project introduced by Jiande Aviation Town in recent years allows the tourists to jump from an altitude of 4,000 meters and experience the thrilling feeling of friction with the air when descending at a high speed and the feeling of flying in the blue sky. This thrilling experience of intimate contact with the sky, and then falling suddenly will become a unique memory that participants will never forget. Jiande launched the helicopter diving project which becomes the first "low-altitude tourism + helicopter diving" project in the country on July 30, 2019.

2.2.2 Experience of amusement events. The project experience provided by Jiande Aviation Town for tourists on the amusement project puts teaching into 
amusement, which also achieved great results. Fulinshan Automobile Park is a car theme park built to meet the needs of tourists for racing and experience extreme speeds. It is also the designated training base for the China Car Drift Championship. The park regularly holds racing races and trick performances to show visitors and players the charm of racing. The main items of the auto park are karts, racing drifts and off-road vehicles. There are different models of karts for players to choose. Players can choose the model that suits them to take part in the race, and whether racing power or drag racing speed is enough for players to experience the excitement of their own cars, racing on a truly professional racetrack and unleash player's potential. The car drift is divided into two parts: the car drift race and the drift experience. The car drift is completed by the professional racers on the track, and the player who win the highest score is selected for the champion. The drift experience is a public-facing project which the professional racing driver takes the tourists sitting on the co-pilot to experience the speed of the car, the excitement of drifting and the thrill of extreme sports. The theme park organizes sports competitions and integrates leisure and entertainment with cultural creativity so that young people can not only learn a lot of detailed car culture, but also personally contact, brain-design and even hand-made models and components. The adventurous spirit of teenagers has also improved their practical ability.

\subsubsection{Hotel catering culture and leisure health} experience. Jiande Aviation Town has a unique hotel catering culture which fully meets the needs of tourists for food and accommodation. The overall theme of the Cloud Aviation theme hotel is the design of the space capsule and boarding platform, which provides tourists with food named Organic fish heads, one of Zhejiang's famous dishes and beverages. The chefs in the hotel will make the organic fish into fresh and nutritious soup which brings tourists different taste experiences and varied Jiande flavors with the delicious spicy and tender fish heads. The Flora Aviation theme restaurant officially opened to the public at the end of June 2018 and located in the core area of Jiande Aviation Town. The prototype of the restaurant is a BAe146-300 passenger transport aircraft which has been transformed into a special aviation theme. The restaurant is mainly engaged in western food, and the open-air area meets the barbecue function. The restaurant not only provides different levels of capsule areas for tourists to choose but also provides 3D driving simulation experience services for tourists. Every detail can provide tourists with a real lift and flight experience and also a great sense of experience. These facilities have become the basis for Jiande Aviation Town to attract more passenger traffic and are some of the advantages of Jiande Aviation Town. Jiande Aviation Town provides leisure and health programs for tourists and forms a unique hot spring health culture. The water in the town hot spring comes from the depth of 1,406 meters and contains extremely rich trace elements that are beneficial to the human body and hydrogen sulfide. It is a health resort for leisure and entertainment with a pure natural high-quality sulfur hot spring which can not only promote blood circulation but also melt away the excess fat of human body and achieve the effect of easy weight loss. As a gift from nature, the quality of the spring water in the aviation town hot spring is natural and pure, and it is also the ingenuity of the designers and builders of the aviation town forming the unique advantages of the Jiande aviation town. The hot spring project has perfected the supporting resources of special dining and entertainment in the aviation town, which is the ingenuity of the aviation town tourism. In addition, Jiande Aviation Town also provides a set of slowmoving living systems for tourists. This system promotes people to maintain health, travel slowly and fully enjoy the "slow" healthy lifestyle. Slow walking can not only release the pressure of life for tourists but also truly enjoy life and make life more poetic.

\subsection{Aviation virtual experience and popular science education block}

The service experience of the aviation town refers to the demand of tourists for the project or the experience of the aviation town. People's living and consumption levels are constantly improving, and people pay more attention to spiritual satisfaction and enjoyment in the new era. The effectively improvement of the psychological satisfaction and identity of tourists asks Jiande Aviation Town to establish and improve the corresponding service system. Jiande Aviation Town combines the service system with the aviation industry chain to comprehensively improve the town's service experience and make each design of the product and project more humane to make service experience more dominant.

\subsubsection{Aviation virtual experience block. Jiande} Aviation Town provides tourists with aviation experiential services and the education of aerospace science and technology for teenagers to learn aviation knowledge and skills. The flight simulation experience is a very unique activity provided by Dachuan Aviation Patriotic Education Base in Jiande Aviation Town as a course. Jiande Aviation Town has the largest quasiprofessional flight simulation hall in China with an area of about $300 \mathrm{~m}^{2}$ and has introduced science-level and quasi-professional advanced flight simulators. Virtual flight is carried out according to the procedures as the following four steps: First, professional flight technicians will explain the flight principle knowledge when students participating in simulated flight so that students can master the basic flight principles and structure. Then perform basic flight operations. After the basic flight, the trainee must complete the challenges of flight tasks such as take-off, cornering and landing. Finally, perform a full-motion flight simulator experience with trainer to experience bumps and taxis during day and night flight and emergency handling of different problems and operations. The virtual flight experience allows students to release their nature, learn real flight knowledge and make students have a strong interest in aviation and sports while improving their diathesis. While enabling 
people to develop in an all-round way, students can get a closer understanding of the pilot's work and life through basic operations, feel more real flight fun in a virtual environment and get a different initial flight experience in practice.

\subsubsection{Aviation popular science education} experience block. Jiande Aviation Town provides aviation science education blocks, cultivates students' hobbies or interests in aviation and establishes great aviation dreams, thereby promoting the development of China's space industry and cultivating more aviation talents. The aviation theme park of Jiande Aviation Town is a "tourism + science" model which includes the Aviation Expo Hall, Aviation Science and Technology Museum and the relevant knowledge of tandem aviation science and technology demonstration experience. And the park shows tourists the development of Jiande Aviation Town so that tourists can better understand the aviation industry. The museum also provides interactive experience technology equipment for tourists to have more interesting interaction during learning a sense of experience, increase interest in learning and get a deeper feel of the unique charm of Jiande Aviation Town. Dachuan Aviation Patriotic Education Base is a science education base in Jiande Aviation Town, which was founded by Zhuhai Dachuan Aviation Technology Co. Ltd. The base has a large number of talents, education and technical resources including professional pilots and experts with a lot of flying experience. The base provides a variety of aviation projects such as a traffic command center, aviation safety knowledge courses, pilot physical fitness training and aviation knowledge lecture halls. It can enable young people to understand aviation lifesaving, enhance flight safety awareness and also allow young people to experience the quality and ability of pilot in the training process to cultivate their explosiveness and courage.

\section{The outlook of experiential layout of Jiande Aviation Town}

(1) The experiential value of Jiande Aviation Town depends on the experience of tourists. Jiande Aviation Town not only innovates the experience method but also perfects the aviation experience project. It effectively improves the psychological satisfaction and identification of tourists and fully enhances the aviation experiential value of the town. Innovation is an important driving force for the sustainable development of an industry. Jiande Aviation Town has innovated the way tourists experience, and satisfying tourists' experience is also a way to enhance the competitiveness of the industry.

(2) According to local conditions, Jiande Aviation Town effectively saved costs and improved resource utilization by rationally allocating and reconstructing Qiandao Lake General Aviation Airport, Xin'an Yu Hot Spring and other resources. Tourists feel the local characteristics in the amusement and form a unique experience exclusive to Jiande Aviation Town. That makes Jiande Aviation Town expand its unique advantages and improve its market competitiveness.

(3) The achievements of Jiande Aviation Town are inseparable from national policies and the support of local government. Meanwhile, Jiande Aviation Town has also promoted the local regional economic development and has made an excellent demonstration for the construction of national aviation characteristic towns.

(4) By using MR, the most advanced virtual reality technology now in the world, some environmental information in the real world can be analyzed in the virtual world. The wide application of MR technology in the aviation field and competitive projects can build a bridge for users to feedback and interact between the virtual and real world, which enables users to analyze data and information more quickly and clearly. In addition, MR technology also enhances the experience of users.

\section{Acknowledgments}

This article is the staged result of the 2018 Shenyang City Philosophy and Social Science Planning Project of Liaoning Province, "Shenyang General Aviation Characteristic Cultural Tourism Town Construction Model Study" (project number 18031).

\section{References}

1. Gao, J., Li, Y., Chen, D. (2019) Research on the Development Status and Standard System Construction of Aviation Towns. J. China Standardization, 03: 79-83.

2. Zhang, H. (2019) Development Strategy of Lowaltitude Tourism Projects in China from the Perspective of Global Tourism. J. Air Transport Business, 01: 49-52.

3. Wang, J. (2019) On the Planning and Design of Contemporary Navigation Town. J. Urban Architecture, 16: 122-125.

4. Xin, J., Song, X., Sha, P. (2019) Comprehensive Evaluation of the Niche of Chinese Characteristic Small Towns: Taking Hangzhou Characteristic Towns as Examples. J. Research World, 09: 3-9.

5. Wang, S., Zhu, H. (2019) An Innovative Study on Tourism Experience in Jingchu's Characteristic Towns-Taking Ai Feike Town as an Example. J. Enterprise Technology and Development, 01: 76-77. 